TITLE:

\title{
Efficacy of nondrug lifestyle measures for the treatment of nocturia.
}

\section{$\operatorname{AUTHOR}(\mathrm{S}):$}

Soda, Takeshi; Masui, Kimihiko; Okuno, Hiroshi;

Terai, Akito; Ogawa, Osamu; Yoshimura, Koji

\section{CITATION:}

Soda, Takeshi ... [et al]. Efficacy of nondrug lifestyle measures for the treatment of nocturia.. The Journal of urology 2010, 184(3): 1000-1004

\section{ISSUE DATE:}

2010-09

URL:

http://hdl.handle.net/2433/129437

\section{RIGHT:}

(c) 2010 American Urological Association Education and Research, Inc. Published by Elsevier Inc.; This is not the published version. Please cite only the published version.; こ の論文は出版社版でありません。引用の際には出版社版をご確認ご利用ください。 
Efficacy of Non-Drug Lifestyle Measures for the Treatment of Nocturia

Takeshi Soda, Kimihiko Masui, Hiroshi Okuno, Akito Terai,

9 Department of Urology, Kurashiki Central Hospital, Okayama, Japan $10(T S, A T)$

11 Department of Urology, Kyoto Medical Center, Kyoto, Japan (KM, HO) 12 Department of Urology, Kyoto University Graduate School of Medicine, 13 Kyoto, Japan (OO, KY)

tCorresponding author. Department of Urology, Kyoto University

16 Graduate School of Medicine, Shogoin-Kawahara-cho 54, Sakyo-ku, Kyoto

17 606-8507, Japan. Tel: +8175 751 3337; Fax: +8175751 3740

18 E-mail address: ky7527@kuhp.kyoto-u.ac.jp (Koji Yoshimura).

20 Running head:

21 Lifestyle modifications for nocturia

Keywords:

24 Lifestyle modification

25 Nocturia

26 Quality of life

29 Word count of text: 2123

31 Word count of the abstract: 249 
ABSTRACT

Purpose: Nocturia has a major impact on quality of life and affects numerous aspects of health. Lifestyle modifications are expected to be helpful in improving nocturia; however, the efficacy of this

38 strategy has not been established. The aim of this study is to test

39 the efficacy of non-drug lifestyle measures as a first step to treat nocturia and to find factors predictive of the efficacy of the

41 intervention.

42 Materials and Methods: We conducted a prospective evaluation on 56

43 patients treated at three hospitals between 2005 and 2009 for

44 symptomatic nocturia. The patients were advised to modify their

45 lifestyle in order to improve nocturia. Lifestyle modifications

46 consisted of four directives - restriction of fluidintake, refraining

47 from excess hours in bed, moderate daily exercise and keeping warm

48 in bed. The frequency volume chart, International Prostate Symptom 49 Score and Pittsburgh Sleep Quality Index before and 4 weeks after 50 the intervention were used to evaluate the efficacy of the therapy.

51 Results: The mean number of nocturnal voids and the nocturnal urine 52 volume significantly decreased from 3.6 to 2.7 ( $p<0.0001$ ) and from $53923 \mathrm{ml}$ to $768 \mathrm{ml}(p=0.0005)$, respectively. Of the 56 patients, 26 54 (53.1\%) showed an improvement of more than one episode. This treatment 55 was significantly more effective in patients with a larger amount 
56 of 24 h urine production.

57 Conclusions: Non-drug lifestyle measures were effective in reducing

58 the number of nocturia episodes and improving patients' quality of

59 life. Patients with polyuria showed a better response to the treatment. 


\section{Introduction}

61

62 Nocturia has a major impact on quality of life, affecting numerous

63 aspects of health, contributing to fatigue, memory deficits,

64 depression, increased risk of heart disease, and gastrointestinal

65 disorders. ${ }^{1-4}$ Nocturnal or global polyuria, reduced nocturnal bladder

66 capacity and sleep disturbance are the possible causes of nocturia. ${ }^{5,6}$

67 Medical therapy with anticholinergic agents, desmopressin and time

68 release diuretics is often considered as first line treatment

69 depending on the diagnosis and underlying cause; however, medication

70 has a potential risk of adverse events and would also cause problems

71 with national medical expenditures as the number of patients increase

72 as a result of aging of the population. Lifestyle modifications such

73 as fluid restriction and sleep enhancement are expected to be

74 helpful, ${ }^{1,2,7}$ but effectiveness of this strategy has not been

75 established. The aim of this study was to test the efficacy of non-drug

76 lifestyle measures as a first step to treat nocturia. We also sought

77 to find factors predictive of the efficacy of the intervention. 
Materials and methods

81 Of the patients who visited our outpatient clinic with a chief

82 complaint of nocturia, those with nocturnal frequency of two or more

83 voids were enrolled in this study. Exclusion criteria were post void

84 residual volume of more than $50 \mathrm{ml}$, untreated urogenital malignancies

85 or renal, cardiac or hepatic failure. The patients who strongly sought

86 medical treatment were also excluded.

After completing a $72 \mathrm{~h}$ frequency volume chart (FVC), the patients

were advised to modify their lifestyle in order to improve nocturia.

89 Lifestyle modifications consisted of four directives - restriction

90 of fluid intake, refraining from excess hours in bed, moderate daily

91 exercise and keeping warm in bed. We paid attention to avoiding

92 imposing too strict measures on the patients, and gave them an

93 explanatory brochure to aid their understanding of the procedure.

94 In the brochure we said, "Examples of lifestyles that can be associated

95 with nocturia are listed below. If you think one or more are applicable

96 to you, please follow the appropriate recommendations." For fluid

97 restriction, we instructed the patients that daily fluid intake of

$982 \%$ of the body weight (i.e., $1000 \mathrm{ml}$ for a person weighing $50 \mathrm{~kg}$ )

99 is enough. We also told patients to restrict fluid in particular in 100 the evening and to avoid excess alcohol or caffeine intake. Secondly, 101 we explained that the patients should not stay in bed for a long time 
102 and that excess bedtime hours would make their sleep shallower, 103 leading to worsening of the nocturia. Thirdly, as an example of 104 moderate daily exercise, we told the patients "If you can walk, try 105 to walk 20 minutes a day. Walking in the evening would be more 106 effective." Finally, we suggested taking a hot water bottle to bed 107 as one of the methods to keep warm in bed.

108 The FVC, International Prostate Symptom Score (IPSS) ${ }^{8}$ and Pittsburgh 109 Sleep Quality Index (PSQI) ${ }^{9,10}$ before and 4 weeks after the intervention 110 were used to evaluate the efficacy of the therapy. The Japanese 111 versions of these questionnaires have been validated previously. ${ }^{8,10}$ 112 Outcomes were defined as excellent (nocturia decreased by $\geq 2$ episodes 113 / night), improved (nocturia decreased by $\geq 1$ episode / night), or 114 unchanged. We offered further medical therapy to the non-responders 115 The PSQI is a self-rated questionnaire for evaluating subjective sleep 116 quality. The questions are combined to obtain a global score ranging 117 from 0-21, with higher scores indicating worse sleep quality. The 118 global score of $>5$ is considered to indicate a sleep disorder. ${ }^{9}$ 119 Nocturnal urine volume (NUV) was defined as the total volume of 120 urine passed during the night including the first morning void. ${ }^{11}$ 121 Nighttime was defined as the period between going to bed with the 122 intention of sleeping and waking with the intention of rising. ${ }^{12}$ From 123 the FVC variables, the following measurements were derived as 124 described elsewhere: ${ }^{13}$ the nocturnal polyuria index (NPi: the ratio 
125 of NUV to 24 h urine volume); the nocturia index (Ni: a measure of 126 nocturnal urine overproduction; a higher score indicating a greater 127 nocturnal urine overproduction); the predicted number of nocturnal 128 voids (PNV); and the nocturnal bladder capacity index (NBCi:

129 reflective of nocturnal bladder capacity, a higher score suggesting 130 diminished NBC). The nighttime to daytime diuresis ratio was

131 calculated as [NUV(ml) / nighttime(h) ] / [(24h urine volume - NUV) 132 (ml) / (24-nighttime) (h)] .

133 For statistical analyses, a Wilcoxon signed rank test was used to 134 compare repeated measurements of variables. Spearman correlation 135 coefficients were used to examine the relationship between baseline 136 parameters and the degree of improvement (change in nighttime 137 frequency). Nominal data were analyzed using the Fisher's exact test. 138 Results were considered significant at $p<0.05$. Statistical analysis 139 was performed using GraphPad Prism®, version 5.

Results

143 Data from 56 patients (47 men and nine women) were evaluated. The 144 mean age was $74.5 \pm 5.7$ years (range 59-85) and the mean body mass 145 index was $21.8 \pm 3.2$ (range $15.0-30.9$ ). Underlying medical disorders 146 included hypertension (13 patients, $23.2 \%$ ), diabetes mellitus (12, $14721.4 \%)$, cardiac conditions $(10,17.9 \%)$ and sleep apnea $(3,5.4 \%)$. 
148 Medical prescriptions included alpha-blockers (13, 23.2 \%),

149 cholinergics $(2,3.6 \%)$, anti-cholinergics $(10,17.9 \%)$ for lower

150 urinary tract symptoms, and diuretics $(5,8.9 \%)$ for cardiac

151 conditions. We did not change the previously prescribed drugs and 152 just added the lifestyle modifications.

153 Abstracts from the FVC before and after the intervention showed 154 significant objective improvement in the symptoms as shown in Table 155 1. The mean number of nocturnal voids and the NUV decreased from 3.6 $156 \pm 1.1$ to $2.7 \pm 1.2 \mathrm{ml}(p<0.0001$, Fig. 1$)$ and from $923 \pm 332 \mathrm{ml}$ to $157768 \pm 339 \mathrm{ml}(p=0.0005)$, respectively. The percentage of patients 158 with improved and excellent responses was $53.1 \%$ and $24.5 \%$, 159 respectively. The $24 \mathrm{~h}$ frequency of micturition also decreased from $16011.6 \pm 2.6$ to $10.7 \pm 2.9$ times $(p=0.0065)$. Analyses of the FVC-derived 161 variables revealed significant improvement in NPi, Ni and NBCi (Table 162 1). The proportion of patients with normal NPi (less than $33 \div{ }^{12}$ ) 163 increased from 4\% to 20\%. The mean nighttime to daytime diuresis ratio 164 decreased from $1.46 \pm 0.38$ to $1.32 \pm 0.43(p=0.0052)$.

165 Patient-reported mean number of nocturia episodes (IPSS question 166 7) and IPSS-QOL (quality of life) score significantly decreased after 167 the intervention (Table 2). In 31 out of the 56 patients (54.4\%), 168 the nocturia improved (decreased by $\geq 1$ points). In 28 (50.0\%), the 169 IPSS-QOL score improved by 1 or more points. There were no significant 170 changes in the other scores (IPSS 1 through 6) before and after the 
171 intervention. Analyses of the PSQI revealed that although the PSQI

172 global score did not show a significant change, the sleep quality

173 score significantly improved after the intervention.

174 Of the non-responders $52 \div(13 / 25)$ underwent medical therapy,

175 which included anti-cholinergics, desmopressin, diuretics, or

176 non-steroidal anti-inflammatory drugs. Other non-responders

177 continued the lifestyle modifications instead of taking medicine.

178 Among the baseline parameters tested, $24 \mathrm{~h}$ and nocturnal urine

179 volume were significantly associated with the degree of improvement

180 (Fig. 2). In addition, 10 patients (40\%) out of 25 presenting with

181 a larger volume of $24 \mathrm{~h}$ urine production (greater than the median

182 value) achieved an excellent response as compared to only 2 patients

183 ( $8.3 \%$ ) out of 24 with less than the median value (Fisher's exact

184 test $p=0.018$ ). All 12 patients who achieved an excellent response

185 were male and none of the female patients showed an excellent response;

186 however, it is difficult to draw a definite conclusion about the sex

187 difference on the efficacy of the treatment because of the small number

188 of female patients.

189 There was no significant worsening of the health status reported 190 by the patients during this period. 
194 Nocturia is associated with various kinds of medical conditions such

195 as overactive bladder, prostatic disease, diabetes mellitus,

196 cardiovascular disease and sleep disorders. ${ }^{1}$ Therapeutic strategies

197 include reducing NUV, increasing nocturnal bladder capacity and

198 treating sleep disorders. Lifestyle modification or behavioral

199 therapy are often mentioned in the literature, mainly in an attempt

200 to reduce nocturnal urine volume; however, their efficacy has not

201 been established and some authors believe that these treatments are 202 of limited efficacy and that pharmacologic therapy is the only option 203 for the majority of patients. ${ }^{6}$ In this study, we attempted to evaluate 204 the efficacy of this strategy by combining four types of lifestyle 205 modifications.

206 The first step in our treatment is fluid restriction. Many people, 207 encouraged by articles in the media, believe that a high water intake 208 will help to prevent ischemic heart disease or cerebrovascular disease 209 and they indeed drink a lot of water; however, there is no definite 210 evidence that stroke or myocardial infarction can be prevented by 211 increasing the water intake in daily life, and that a high intake 212 of water will actually decrease blood viscosity. ${ }^{14}$ Reducing fluid 213 intake has already been recommended in patients whose nocturia is 214 secondary to nocturnal polyuria; ${ }^{1,15}$ however, there has been no 215 recommendation stating the actual means of fluidrestriction. As total 21624 h urine volume in healthy individuals was reported to be $23.0 \pm$ 
$2171.7 \mathrm{ml} / \mathrm{kg}^{16}$ and this is roughly equivalent to drinking fluid of $2-2.5 \%$

218 of body weight, we made a recommendation as described in the Methods

219 section.

220 Second, we recommended shortening the time spent in bed to improve

221 sleep quality. We previously reported that patients with nocturia

222 spent a significantly longer time in bed than controls. ${ }^{17,18}$

223 The third measure was to maintain moderate daily exercise. Asplund

224 and Aberg reported that lack of regular exercise was associated with

225 an increased number of nocturnal micturition episodes, ${ }^{19}$ and Sugaya

226 et al. showed the efficacy of walking exercise in the treatment of

227 nocturia. ${ }^{20}$ By using the protocol of 30 min rapid walking in the evening

228 or night for 8 weeks, they achieved a significant reduction in the

229 number of nocturia episodes from 3.3 to 1.9. It is a very useful

230 behavioral therapy; however, we were afraid that some of the elderly

231 patients would find difficulty in carrying out this practice. In order

232 to make the protocol suitable for as many patients as possible

233 including those with impaired activities of daily living, we made

234 a recommendation of moderate exercise, where possible, as described

235 in the Methods section.

236 Finally, we included a recommendation to keep warm in bed. Cold

237 exposure is known to increase urine output by inducing "cold diuresis"

238 through a combination of increased venous return, activation of atrial

239 natriuretic peptide, decreased levels of antidiuretic hormone and 
renal antidiuretic hormone receptor, and tubular dysfunction. ${ }^{21,22}$ In

241 our previous study using a self-reported questionnaire on the reasons

242 for change in night time frequency in patients with symptomatic

243 nocturia, feeling cold in bed was the third most prevalent answer

244 next to increased fluid intake and longer duration in bed. ${ }^{18}$ We have

245 also shown that urinary symptoms including nocturia worsen in winter

246 in the general population. ${ }^{23}$

247 The major limitation of our study is the lack of a control group.

248 Although the gold standard for study design is a randomized controlled

249 trial, it is difficult to set a control group in this kind of study.

250 A possible placebo effect and a patient selection bias should

251 therefore be kept in mind in interpreting the results. Nevertheless,

252 the objective improvements shown in the FVC analyses strongly support

253 the efficacy of the therapy. Another weakness of the study is that

254 we did not structurally obtain the information about the degree to

255 which the patients actually incorporated the behavioral

256 recommendations. Although that is an important issue, we did not want

257 to frustrate the patients by burdening them with writing down the

258 details of their lifestyle in addition to keeping the FVC and answering

259 the questionnaires. We decided that the IPSS, PSQI and FVC were the

260 minimal requirements to evaluate the efficacy of our therapy. It is

261 therefore difficult to assess which component worked best for each

262 patient. The most effective and suitable intervention to the given 
baseline conditions should be determined as the next step. Finally,

264 the long-term effect remains to be confirmed. This point should also

265 be clarified in the future studies.

266 In conclusion, the present study demonstrated that lifestyle

267 measures were effective in improving nocturia, and patients with

268 larger 24 h urine volume showed a better response. Assessment of FVC

269 and FVC-derived variables revealed that the number of nocturnal voids

270 and nocturnal urine volume significantly decreased, and NPi, Ni and

271 NBCi significantly improved after the intervention; however, bladder

272 capacity did not show any significant change. This implies that the

273 efficacy of this therapy is primarily attributable to the reduction

274 of nocturnal urine volume.

\section{Conclusions}

278 Non-drug lifestyle measures were effective in reducing the number 279 of nocturia episodes and improving patients' QoL. The effect of this 280 therapy was shown to result from a reduction in NUV. Patients with 281 polyuria showed a better response to the treatment.

\section{Abbreviations}

285 FVC: frequency volume chart 
286 IPSS: International Prostate Symptom Score

287 NBCi: nocturnal bladder capacity index

288 Ni: nocturia index

289 NPi: nocturnal polyuria index

290 NUV: nocturnal urine volume

291 PNV: predicted number of nocturnal voids

292 PSQI: Pittsburgh Sleep Quality Index

293 


\section{REFERENCES}

295 1. Weiss JP and Blaivas JG: Nocturia. J Urol 2000;163:5.

296 2. Marinkovic SP, Gillen LM and Stanton SL: Managing nocturia. BMJ $2972004 ; 328: 1063$.

298 3. Asplund R: Nocturia in relation to sleep, somatic diseases and 299 medical treatment in the elderly. BJU Int 2002;90:533.

300 4. Asplund $R$ and Aberg $H$ : Health of the elderly with regard to sleep 301 and nocturnal micturition. Scand J Prim Health Care 1992;10:98. 302 5. Weiss JP and Blaivas JG: Nocturnal polyuria versus overactive 303 bladder in nocturia. Urology 2002;60:28.

304 6. Appell RA and Sand PK: Nocturia: etiology, diagnosis, and treatment. 305 Neurourol Urodyn 2008;27:34.

306 7. Weatherall M and Arnold T: Nocturia in adults: draft New Zealand 307 guidelines for its assessment and management in primary care. N Z 308 Med J 2006;119:U1976.

309 8. Homma Y, Tsukamoto T, Yasuda K et al: Linguistic validation of 310 Japanese version of International Prostate Symptom Score and BPH 311 Impact Index Score. Nippon Hinyokika Gakkai Zasshi 2002;93:669

312 9. Buysse DJ, Reynolds CF, Monk TH et al: The Pittsburgh Sleep Quality 313 Index: a new instrument for psychiatric practice and research.

314 Psychiatry Res 1989;28:193.

315 10. Doi Y, Minowa M, Uchiyama M et al: Psychometric assessment of 316 subjective sleep quality using the Japanese version of the Pittsburgh 
317 Sleep Quality Index (PSQI-J) in psychiatric disordered and control 318 subjects. Psychiatry Res 2000;97:165.

319 11. van Kerrebroeck P, Abrams P, Chaikin D et al: The standardisation 320 of terminology in nocturia: report from the standardisation

321 Sub-committee of the International Continence Society. Neurourol 322 Urodyn 2002;21:179.

323 12. Van Kerrebroeck P, Abrams P, Chaikin D et al. The standardization 324 of terminology in nocturia: report from the standardization 325 subcommittee of the International Continence Society. BJU Int 2002;90 326 Suppl 3:11.

327 13. Weiss JP, Blaivas JG, Stember DS et al: Evaluation of the etiology 328 of nocturia in men: the nocturia and nocturnal bladder capacity 329 indices. Neurourol Urodyn 1999;18:559.

330 14. Sugaya K, Nishijima S, Oda M et al: Change of blood viscosity 331 and urinary frequency by high water intake. Int J Urol 2007;14:470. 332 15. Reynard J: Fluid balance therapy of nocturia in women. Int 333 Urogynecol J Pelvic Floor Dysfunct 1999;10:43.

334 16. Matthiesen TB, Rittig S, Nørgaard JP et al: Nocturnal polyuria 335 and natriuresis in male patients with nocturia and lower urinary tract 336 symptoms. J Urol 1996;156:1292.

337 17. Yoshimura $\mathrm{K}$ and Terai A: Classification and distribution of 338 symptomatic nocturia with special attention to duration of time in 339 bed: a patient-based study. BJU Int 2005;95:1259. 
340 18. Yoshimura $K$ and Terai A: Fluctuation of night time frequency in 341 patients with symptomatic nocturia. Int J Urol 2005;12:469.

342 19. Asplund $R$ and Aberg HE: Nocturia in relation to body mass index, 343 smoking and some other life-style factors in women. Climacteric $344 \quad 2004 ; 7: 267$.

345 20. Sugaya K, Nishijima S, Owan T et al: Effects of walking exercise 346 on nocturia in the elderly. Biomed Res 2007;28:101.

347 21. Polderman KH: Mechanisms of action, physiological effects, and 348 complications of hypothermia. Crit Care Med 2009;37:S186.

349 22. Sun Z, Zhang Z and Cade R. Renal responses to chronic cold exposure. 350 Can J Physiol Pharmacol 2003;81:22.

351 23. Yoshimura K, Kamoto T, Tsukamoto T et al: Seasonal alterations 352 in nocturia and other storage symptoms in three Japanese communities. 353 Urology $2007 ; 69: 864$. 


\section{Figure Legends}

358 Fig. 1 - Box plots showing nocturnal frequency before and after the 359 intervention. Whiskers: 10-90 percentiles; $p<0.001$.

360 Fig. 2 - The relationship between pre-treatment $24 \mathrm{~h}$ urine volume

361 (A) and nocturnal urine volume (B) and decreased number of nocturia 362 episodes. A: $r=0.3923 ; p=0.0102 ; B: r=0.3088 ; p=0.0466$. 


\section{TABLES}

Table 1 - Results of frequency volume chart before and after the intervention

\begin{tabular}{|c|c|c|c|c|c|c|}
\hline Variables & & Before & & After & & $p$ \\
\hline \multicolumn{7}{|l|}{ NUV, ml } \\
\hline Mean (SD) & 923 & $(332)$ & 768 & (339) & & \\
\hline Median (range) & 917 & $(235-1650)$ & 707 & $(175-1513)$ & $<$ & 0.001 \\
\hline \multicolumn{7}{|l|}{$24 \mathrm{~h} \mathrm{UV}, \mathrm{ml}$} \\
\hline Mean (SD) & 1850 & $(581)$ & 1716 & $(607)$ & & \\
\hline Median (range) & 1835 & $(665-3537)$ & 1705 & $(675-3084)$ & & 0.024 \\
\hline \multicolumn{7}{|c|}{ Nocturnal frequency } \\
\hline Mean (SD) & 3.6 & $(1.1)$ & 2.7 & $(1.2)$ & & \\
\hline Median (range) & 3.6 & $(2.0-6.5)$ & 2.8 & $(0.3-5.0)$ & $<$ & 0.001 \\
\hline \multicolumn{7}{|l|}{$24 \mathrm{~h}$ frequency } \\
\hline Mean (SD) & 11.6 & $(2.6)$ & 10.7 & $(2.9)$ & & \\
\hline Median (range) & 11.3 & $(6.3-19.5)$ & 10.0 & $(5.3-18 \cdot 5)$ & & 0.007 \\
\hline \multicolumn{7}{|l|}{ MVV, ml } \\
\hline Mean (SD) & 309 & $(99)$ & 313 & $(103)$ & & \\
\hline Median (range) & 300 & $(50-530)$ & 300 & $(50-600)$ & & 0.65 \\
\hline \multicolumn{7}{|l|}{ NPi } \\
\hline Mean (SD) & 50.5 & $(12.2)$ & 45.9 & $(14.7)$ & & \\
\hline Median (range) & 48.3 & $(25.2-75.4)$ & 45.0 & $(14.6-72.6)$ & & 0.004 \\
\hline \multicolumn{7}{|l|}{$\mathrm{Ni}$} \\
\hline Mean (SD) & 3.1 & $(0.9)$ & 2.6 & $(0.9)$ & & \\
\hline Median (range) & 2.9 & $(1.4-5.4)$ & 2.6 & $(1 \cdot 2-4 \cdot 3)$ & $<$ & 0.001 \\
\hline \multicolumn{7}{|l|}{$\mathrm{NBCi}$} \\
\hline Mean (SD) & 1.5 & $(0.7)$ & 1.3 & $(0.6)$ & & \\
\hline Median (range) & 1.6 & $(0.2-3.7)$ & 1.2 & $(0.1-2.8)$ & & 0.002 \\
\hline
\end{tabular}


N/D diuresis ratio

\begin{tabular}{lccccc} 
Mean (SD) & 1.46 & $(0.38)$ & 1.32 & $(0.43)$ \\
Median (range) & 1.44 & $(0.64-2.52)$ & 1.31 & $(0.58-2.19)$ & 0.005 \\
\hline NUV = nocturnal urine volume; MVV = maximum voided volume; NPi \\
= nocturnal polyuria index; Ni = nocturia index; NBCi=nocturnal \\
bladder capacity index
\end{tabular}




\section{TABLES}

Table 2 - Results of IPSS and PSQI before and after the intervention

\begin{tabular}{|c|c|c|c|}
\hline Variables & Before & After & $\mathrm{p}$ \\
\hline \multicolumn{4}{|l|}{ IPSS - 7} \\
\hline Mean (SD) & $3.8 \quad(0.8)$ & $3.1 \quad(1.2)$ & \\
\hline Median (range) & $4.0 \quad(2.0-5.0)$ & $3.0 \quad(1.0-5.0)$ & $<0.001$ \\
\hline \multicolumn{4}{|l|}{ IPSS - QOL } \\
\hline Mean (SD) & $4.6(1.0)$ & $3.8 \quad(1.4)$ & \\
\hline Median (range) & $5.0 \quad(2.0-6.0)$ & $4.0 \quad(0.0-6.0)$ & $<0.001$ \\
\hline \multicolumn{4}{|l|}{ PSQIG } \\
\hline Mean (SD) & 6.56 & 5.95 & \\
\hline Median (range) & $6.0 \quad(0.0-14.0)$ & $5.0 \quad(0.0-14.0)$ & 0.25 \\
\hline \multicolumn{4}{|l|}{ sleep quality } \\
\hline Mean (SD) & $2.43 \quad(0.80)$ & $2.10 \quad(0.72)$ & \\
\hline Median (range) & $2.0 \quad(1.0-4.0)$ & $2.0 \quad(1.0-4.0)$ & 0.007 \\
\hline \multicolumn{4}{|c|}{ Time in bed, hours } \\
\hline Mean (SD) & $8.44 \quad(1.1)$ & $8.38 \quad(1.2)$ & \\
\hline Median (range) & $8.5(6.0-10.5)$ & $8.5 \quad(6.2-11.3)$ & 0.99 \\
\hline
\end{tabular}

IPSS = International Prostate Symptom Score; PSQIG = Pittsburgh

Sleep Quality Index global score 


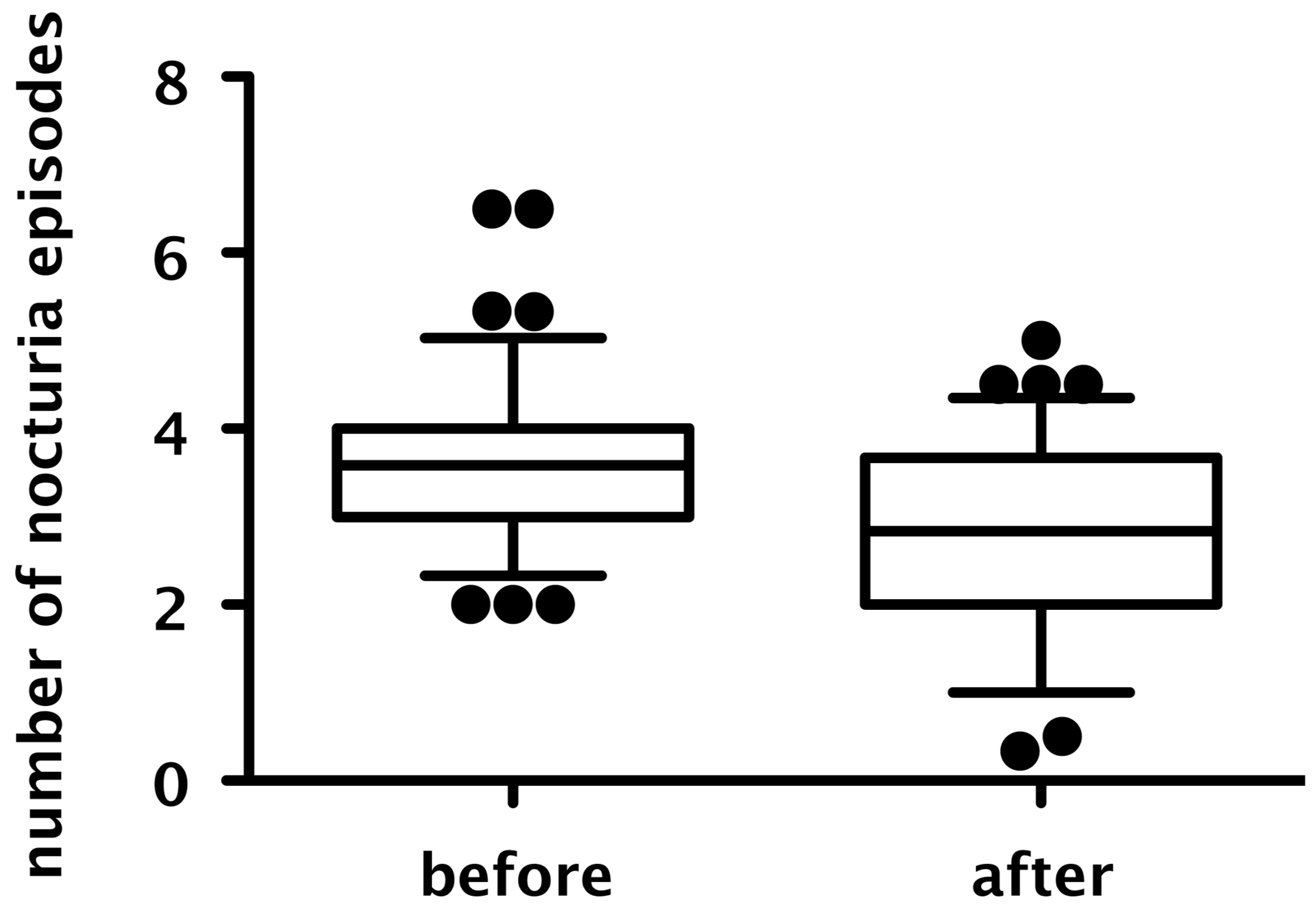




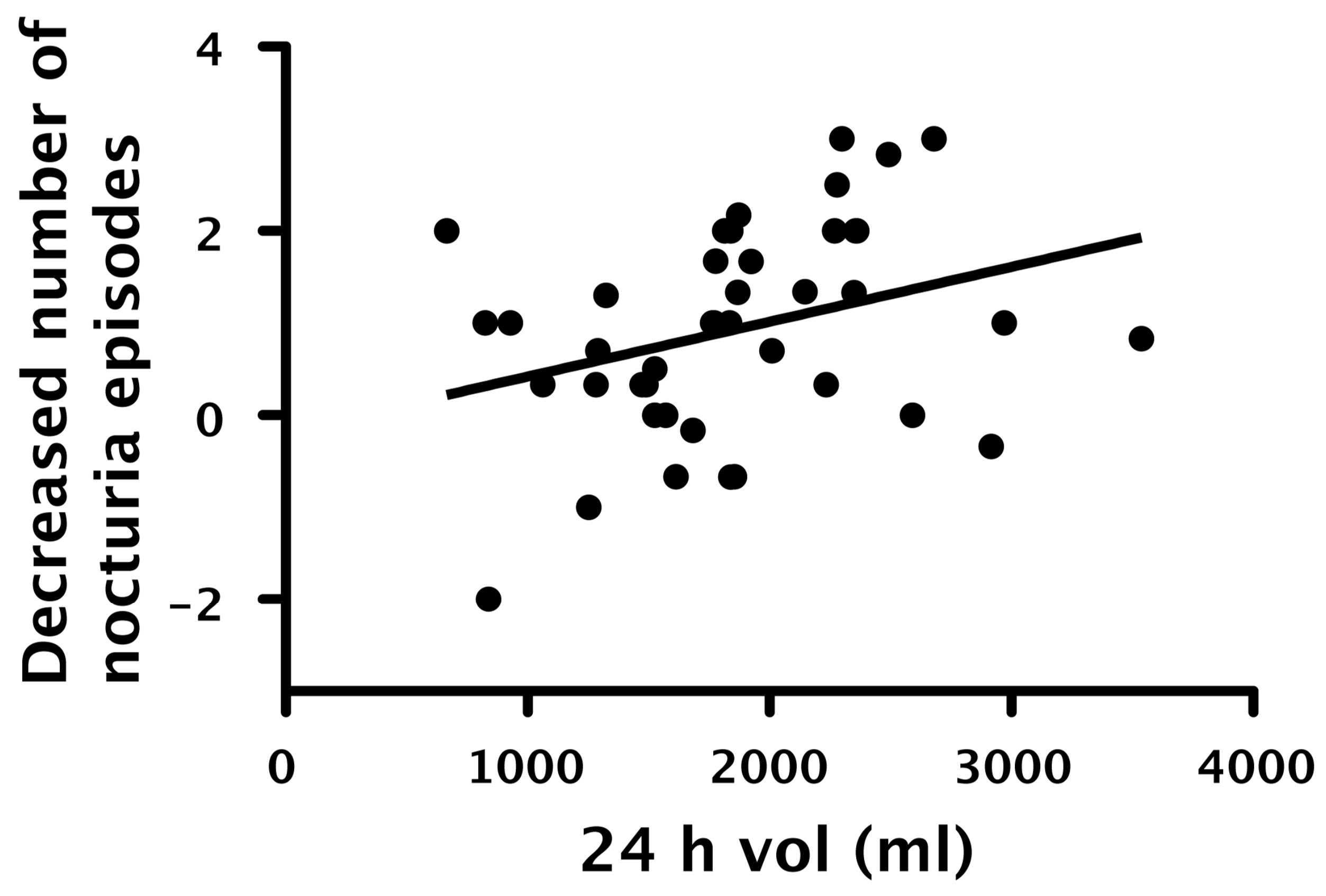




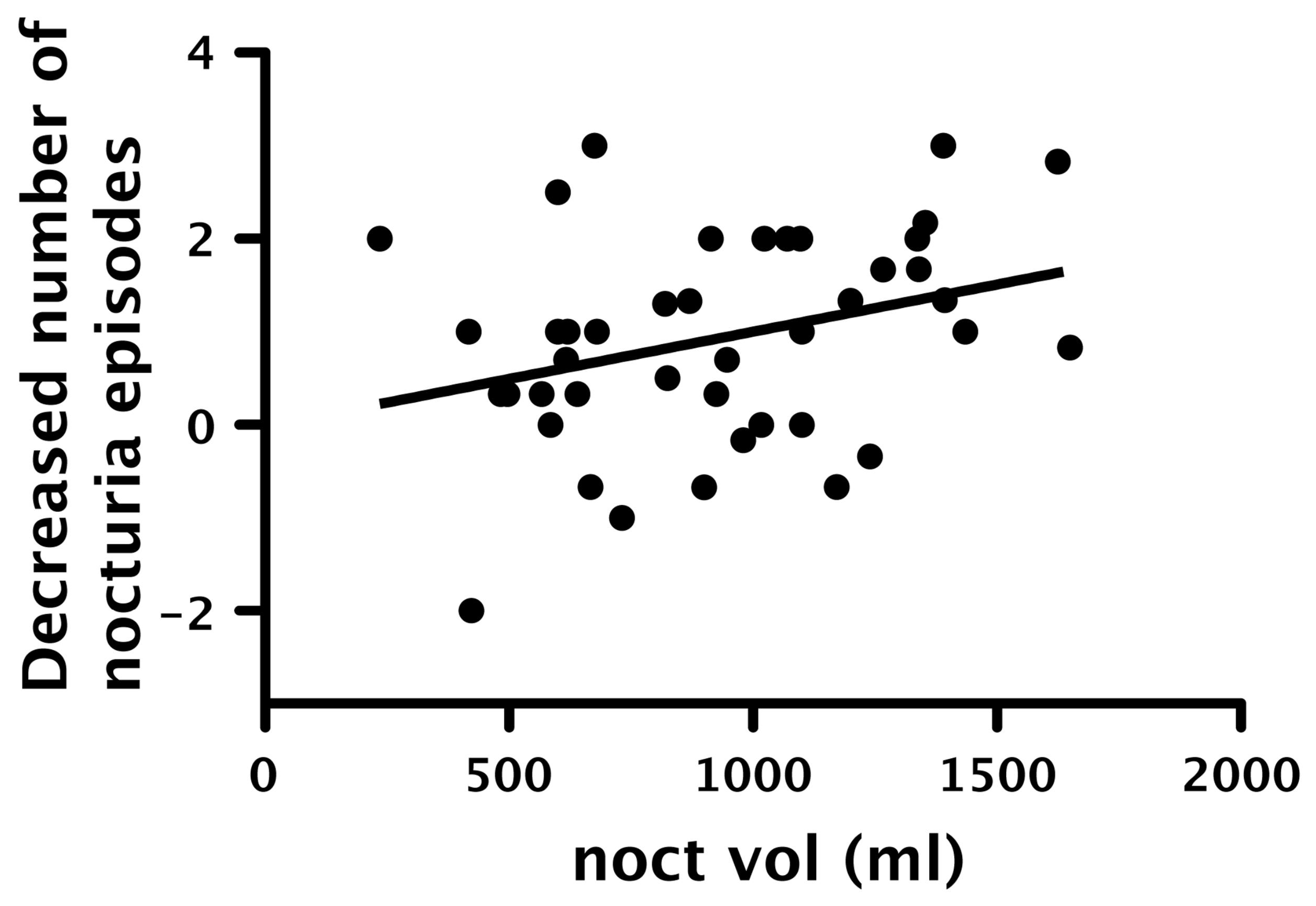

